\title{
NUEVAS ESPECIES DE VIGUIERA (COMPOSITAE, HELIANTHEAE) DEL ESTADO DE DURANGO, MÉXICO'
}

\author{
M. Socorro González Elizondo² \\ Martha GonzÁlez ElizONDO² \\ CIIDIR-IPN Unidad Durango \\ Apartado postal 738 \\ 34000 Durango, Dgo. \\ e-mail: sgonzalez@omanet.com.mx \\ Y \\ JERZY RZEDOWSKI \\ Instituto de Ecología \\ Centro Regional del Bajío \\ Apartado postal 386 \\ 61600 Pátzcuaro, Michoacán
}

\begin{abstract}
RESUMEN
Viguiera oligantha y $V$. inegii se describen como nuevas especies y se ilustran. Ambas son arbustos canescentes que se encuentran en forma simpátrica, endémicos del noreste del estado de Durango. La primera representa una condición poco usual en el género debido a sus cabezuelas notoriamente reducidas, con 1 ó 2 flores del disco y 0 a 2 flores liguladas. Viguiera oligantha y $V$. inegii parecen ser especies hermanas posiblemente relacionadas con V. greggii (A. Gray) S. F. Blake, de la que difieren en tener hojas predominantemente alternas con nervaduras impresas, así como cabezuelas y flores más pequeñas. Ambas especies son afines a los componentes de la serie Brevifoliae, aunque para integrar en ella a $V$. oligantha es preciso ampliar la circunscripción del grupo. Se presenta una clave para la identificación de las especies que conforman la serie Brevifoliae en su carácter enmendado y se incluye una lista de materiales de herbario revisados de los restantes componentes de esa serie.
\end{abstract}

\section{ABSTRACT}

Viguiera oligantha and $V$. inegii, from the state of Durango, are described and illustrated. The two new species are endemic to northeastern Durango and occur sympatrically. Both are canescent shrubs. Viguiera oligantha represents an unusual condition in the genus because of its highly reduced

\footnotetext{
${ }^{1}$ Trabajo desarrollado con apoyo económico de la CEGEPI I.P.N. (Proyecto 990468) y del Instituto de Ecología, A.C. (Cuenta 902-03), así como del Consejo Nacional de Ciencia y Tecnología y de la Comisión Nacional para el Conocimiento y Uso de la Biodiversidad.

2 Becarias de la COFAA y EDI del Instituto Politécnico Nacional.
} 
heads with 1-2 disc flowers and 0-2 ray flowers, the heads arranged in corymbiform cymes. We consider $V$. oligantha and $V$. inegii to be sister species possibly derived from $V$. greggii (A. Gray) S. F. Blake. They differ from $V$. greggii in having predominantly alternate leaves with impressed nerves as well as smaller heads and flowers. The new species are related to members of the series Brevifoliae, although it is necessary to reevaluate the circumscription of the series to incorporate these species. A key is presented for the identification of the components of the emended series Brevifoliae and a list of examined specimens of the remaining species of the series is included.

Colectas del personal del Centro Interdisciplinario de Investigación para el Desarrollo Integral Regional, del Instituto Politécnico Nacional (CIIDIR-IPN) y del Instituto Nacional de Estadística, Geografía e Informática (INEGI) han permitido la detección de dos especies de Viguiera (Compositae, Heliantheae) nuevas para la ciencia. Ambas son arbustos canescentes, se han encontrado conviviendo y son abundantes localmente pero de distribución restringida a una pequeña área del sector noreste del estado de Durango. Tal circunstancia, aunada al hecho de que $V$. inegii ha sido confundida con $V$. greggii (A. Gray) S. F. Blake, ha influido para que estas plantas no hayan sido detectadas con anterioridad como nuevas especies.

Las plantas aquí descritas se conocen únicamente de lomeríos y serranías bajas del área de la cuenca del Río Nazas, donde crecen en laderas y pie de monte con vegetación de matorral xerófilo. Dicha zona forma parte del Desierto Chihuahuense y parece representar una región de concentración de endemismos. Otras especies, en su mayoría plantas arbustivas y subfruticosas que también están restringidas a laderas secas de la cuenca del Nazas, son las siguientes: Henricksonia mexicana B. L. Turner (género monotípico), Cotinus chiangii (Young) Rzedowski \& Calderón, Siphonoglossa durangensis Henrickson \& Hilsenbeck y S. linearifolia Henrickson \& Hilsenbeck. Estos elementos se han registrado de los lomeríos cercanos al Nazas al oriente del estado de Durango, y algunos también de la zona adyacente de Coahuila (Rzedowski y Calderón, 1999; Turner, 1977; Henrickson y Hilsenbeck, 1979). Mammillaria guelzowiana Werdermann se conoce solamente del tipo, de una localidad no indicada, también en las montañas del valle del río Nazas (Bravo y Sánchez Mejorada, 1991). Por otra parte, varias especies encontradas en la cuenca del Nazas, como Bonetiella anomala (I. M. Johnston) Rzedowski, Mimosa setuliseta J. L. Villarreal y Setchellanthus caeruleus Brandegee presentan distribución disyunta, la primera colectada también en San Luis Potosí y Querétaro y la segunda en San Luis Potosí (Villarreal, 1992); mientras que Setchellanthus se conoce del oriente de Durango y del Valle de Tehuacán (Iltis, 1999). Los géneros Cotinus y Setchellanthus representan elementos relictuales (Rzedowski y Calderón de Rzedowski, 1999; Iltis, 1999). La importancia del Desierto Chihuahuense como área de endemismos, particularmente para la familia de las compuestas, ha sido analizada por Rzedowski (1972). Hacia la parte alta de la cuenca del Nazas se distribuyen otras especies con distribución aparentemente endémica: Thymopylla gentryi (M. C. Johnston) Strother y Brongniartia riesebergii O. Dorado, las cuales fueron halladas en terrenos cerriles en los municipios de Rodeo e Indé. Viguiera oligantha y $V$. inegii, aquí descritas, están restringidas a lomeríos secos cercanos al Nazas, en el municipio de Rodeo, al noreste de Durango.

En las descripciones presentadas a continuación, el término "gotas de resina" se refiere a pelos glandulares subsésiles con apariencia de gotas de resina. 
Viguiera oligantha S. González, M. González Elizondo et J. Rzedowski sp. n. Fig. 1.

Fruticosa 0.8-1.4 m alta, ramosissima, ramis junioribus incano-canescentibus; foliis parvis 1-1.8(2.3) cm longis, ovatis obtusis integerrimis vel sublobatis basi cuneatis in petiolum brevem attenuatis infra lanosis albidis supra strigosis; capitulis mediocribus in racemis corymbiformibus aggregatis caulem et ramulos terminantibus; phylariis 1-2(3)serialibus lineari-oblongis usque spathulatis obtusis $2.5-2.8 \mathrm{~mm}$ longis lanati-canescentibus; ligulis $0-2$ oblongis $2.3-2.6 \mathrm{~mm}$ longis luteis pubescentibus, floribus disci $1-2$ corollis $3-3.5 \mathrm{~mm}$ longis luteis puberulis; acheniis glabris; pappo nullo.

Arbusto de 0.8-1.4 m de alto, muy ramificado; tallo y ramas principales con corteza gris-negra longitudinalmente estriada dejando ver la corteza interior de color café-amarillento pálido, ramas superiores delgadas, ascendentes, de color café-amarillento pálido, las jóvenes densamente canescentes con pelos blancos, septados, ascendentes, con gotas de resina amarillas, ramillas claviformes secas a veces presentes (¿restos de ramillas pedunculares?); hojas alternas, ocasionalmente acompañadas por hojas más pequeñas subverticiladas, láminas de las hojas maduras de $1-1.8(2.3) \mathrm{cm}$ de longitud, $0.5-1 \mathrm{~cm}$ de ancho, ovado-deltadas, triangular-deltadas o rómbico-ovadas, enteras a sublobadas en la base, ápice redondeado a obtuso, rara vez subagudo, base cuneada, gradualmente angostada en un pseudopecíolo de $0.5-2 \mathrm{~mm}$ de longitud, superficie conspicuamente reticulada debido a la impresión en el haz y la prominencia en el envés de la nervadura media, las 2(4) nervaduras laterales y las nervaduras secundarias, las nervaduras laterales surgiendo casi desde la base de la lámina, haz de color verde opaco, estrigoso con pelos septados, tuberculados en la base, blancos o translúcidos en la parte inferior y con gotas de resina translúcidas de color amarillo, envés densamente lanoso, de color blanco a gris pálido, estrigoso sobre las nervaduras principales con pelos septados similares a los del haz, la superficie entre las nervaduras secundarias cubierta de pelos blancos o translúcidos más delgados, enmarañados, formando parches entre la reticulación de las venillas, con abundantes gotas de resina; inflorescencias en forma de cimas corimbiformes de 4 a 12 cabezuelas dispuestas al final de las ramillas, de $0.8-1.5 \mathrm{~cm}$ de longitud, $1-2.4 \mathrm{~cm}$ de ancho, extremos pedunculares de las ramillas hasta de $3.7 \mathrm{~cm}$ de longitud; las cabezuelas muy reducidas, de $6-7 \mathrm{~mm}$ de longitud, $2-3.3 \mathrm{~mm}$ de ancho, sobre pedicelos de $0.5-1 \mathrm{~mm}$ de longitud, involucro de 1 a 3 series, de 2.5-3.8 $\mathrm{mm}$ de longitud, 1.5-3.3 $\mathrm{mm}$ de ancho, las brácteas linear-oblongas a espatuladas, obtusas, de 2.5-2.8 mm de longitud, 0.5-0.9 mm de ancho, aplicadas excepto en el ápice, con tres nervaduras visibles en la superficie adaxial que es glabra en la parte inferior, densamente lanoso-canescentes y con gotas de resina en la superficie abaxial, endurecidas y engrosadas (el ápice herbáceo en brácteas jóvenes), disco de aprox. $1 \mathrm{~mm}$ de ancho; flores liguladas 0 a 2, con tubo de 0.6-1.1 mm, lámina de 2.3-2.6 mm de longitud y 1.7-2.6 mm de ancho, tridentadas, con 7 a 9 nervaduras sobre la cara abaxial, amarillas, pubescentes y densamente cubiertas por gotas de resina; flores del disco 1 ó 2, con tubo de 3-3.5 mm de longitud, lóbulos de 0.7-0.8 mm de longitud, reflejos, amarillas, puberulentas, densamente cubiertas por gotas de resina; páleas 1 a 4 , de 2-4.8 mm de longitud, 0.5-1.4 mm de ancho, oblongas, agudas a abruptamente acuminadas, glabras en el lado adaxial, densamente lanosas y con gotas de resina en el abaxial, parcialmente envolviendo a los aquenios; aquenios de las flores liguladas estériles, amarillentos, vilano ausente, los de las flores del disco comprimidos cuando jóvenes, de 


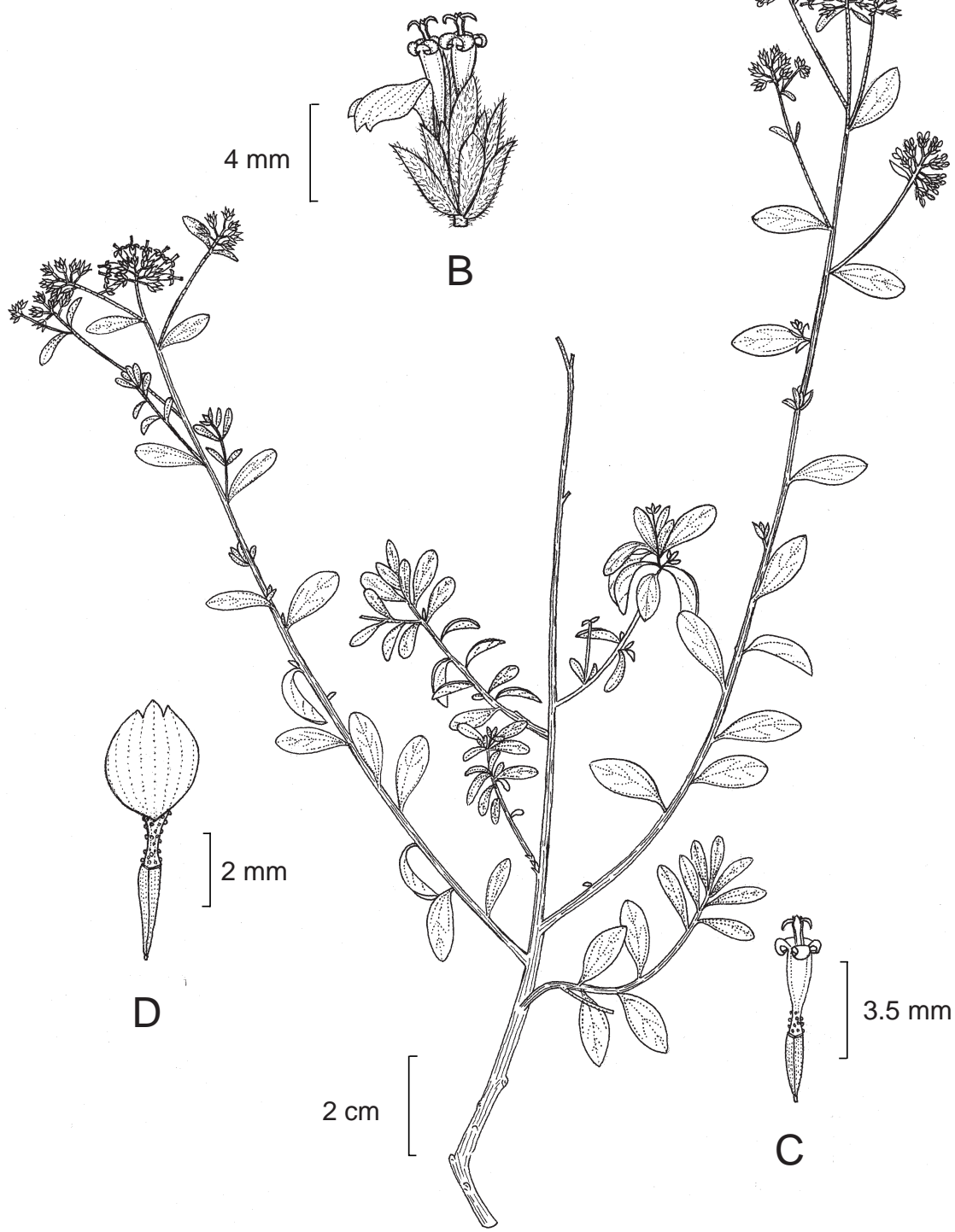

A

Fig. 1. Viguiera oligantha S. González, M. González Elizondo et J. Rzedowski. A. Rama con hojas e inflorescencias; B. Cabezuela; C. Flor del disco, con aquenio fértil; D. Flor ligulada, con aquenio estéril. Dibujo elaborado por Rogelio Cárdenas. 
sección subcuadrangular en la madurez, estriados, de color café obscuro a casi negro, de 2.6-3 $\mathrm{mm}$ de longitud, 0.5-0.8 $\mathrm{mm}$ de ancho, glabros, vilano ausente.

TIPO: México, Durango, $\pm 1 \mathrm{~km}$ al NE de Las Ánimas, sobre la carretera a Nazas, municipio de Rodeo, 2508'36" N, 104²5'59" W, 1340 m, arbusto abundante en pie de monte de lomerío, 5 Ago. 1997, S. González 5909, M. González y S. Acevedo (holotipo: IEB, isotipos: ANSM, CHAPA, CIIDIR, ENCB, MEXU, TEX).

Material adicional revisado: México, Durango, $\pm 1 \mathrm{~km}$ al NE de Las Ánimas, sobre la carretera a Nazas, municipio de Rodeo, 19 Jun. 1997, J. García y V. López (INEGI) 4.3 (CIIDIR).

Esta especie presenta gran similitud con Viguiera inegii, a partir de la cual parece haberse originado en tiempos relativamente recientes mediante la reducción en el número de flores por cabezuela ( 1 ó 2 flores del disco y 0 a 2 flores liguladas) y cambios en el tipo de pubescencia. Las dos se asemejan en los caracteres de hábito, presencia de múltiples ramillas secas café-amarillentas sobresaliendo del follaje, corteza de las ramas similar en color y textura, así como flores tubulares y liguladas de forma, tamaño y color semejantes, ambas con diminutas gotas resinosas. Las principales diferencias entre las dos especies se presentan en el Cuadro 1. En el campo pueden ser claramente separadas por la mayor talla de $V$. inegii (0.8-2 m vs. 0.8-1.4 m), sus hojas de coloración gris más obscura e inflorescencias no cimosas (vs. cimoso-corimbosas). Además, V. oligantha presenta ramas más frágiles y carece de olor desagradable.

Viguiera inegii S. González, M. González Elizondo et J. Rzedowski sp. n. Fig. 2.

Fruticosa 0.8-2 m alta, ramosissima, ramis junioribus incano-canescentibus; foliis parvis (1.3-1.7 cm longis) ovatis rotundatis integerrimis vel sublobatis basi cuneatis in petiolum brevem attenuatis infra strigoso-canescentibus albidis vel cinereis supra strigosis; capitulis plerumque solitariis caulem et ramulos terminantibus; phylariis 2-3-serialibus gradatim imbricatis oblongis obtusis vel acutis strigosis ad basim induratis apice herbaceis; ligulis 2-6 oblongis $3.5-4 \mathrm{~mm}$ longis luteis puberulis, floribus disci $16-45$ corollis luteis puberulis; acheniis 1.9-2.3 mm longis glabris; pappo nullo.

Arbusto de 0.8-2 m de alto, muy ramificado, tallo y ramas principales con corteza gris negra longitudinalmente estriada, dejando ver la corteza interior de color café-amarillento pálido; ramas superiores delgadas, ascendentes, de color café-amarillento pálido, las jóvenes densamente canescentes con pelos blancos, septados, ascendentes, y con gotas de resina amarilla; hojas predominantemente alternas, ocasionalmente acompañadas por hojas más pequeñas subverticiladas o unas pocas opuestas; láminas de las hojas maduras ovado-deltadas, triangular-deltadas o rómbico-ovadas, de $1.3-1.7 \mathrm{~cm}$ de longitud, $0.6-0.9 \mathrm{~cm}$ de ancho, enteras a algo lobuladas, el ápice redondeado, obtuso o subagudo, base cuneada, gradualmente angostada en un pseudopecíolo de 0.5-4 $\mathrm{mm}$ de longitud, superficie conspicuamente reticulada debido a la impresión en el haz y la prominencia en el envés de la nervadura media, 2(4) nervaduras laterales y las nervaduras secundarias, las nervaduras laterales surgiendo casi desde la base de la lámina, haz de color verde opaco, 

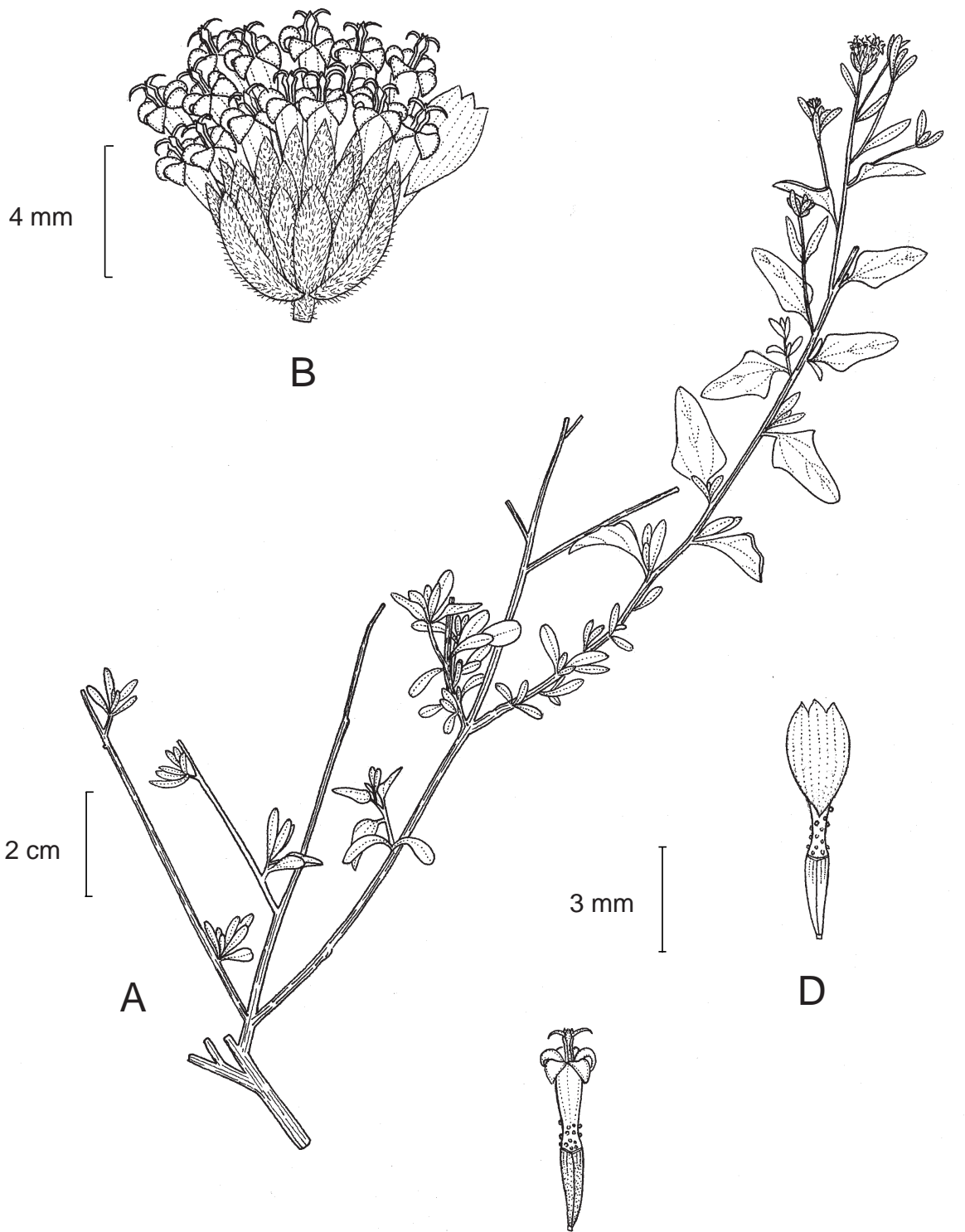

C

Fig. 2. Viguiera inegii S. González, M. González Elizondo et J. Rzedowski. A. Rama con hojas e inflorescencias; B. Cabezuela; C. Flor del disco, con aquenio fértil; D. Flor ligulada, con aquenio estéril. Dibujo elaborado por Rogelio Cárdenas. 
estrigoso con pelos en forma de aguijón, septados, tuberculados en la base, blancos o translúcidos en la parte inferior, de $0.2-0.5 \mathrm{~mm}$ de largo, rectos o muy ligeramente curvados, y con gotas de resina translúcida de color amarillo, envés densamente estrigoso-canescente de color blanco a gris pálido, con pelos similares a los del haz, aplicados y orientados hacia el ápice o los lados de la hoja, y con gotas de resina; cabezuelas solitarias o en grupos de 2 ó 3 sobre pedúnculos de 2-30 mm de largo; cabezuelas de 5-7 mm de longitud y de 7-13 mm de ancho, involucro graduado en 2 a 3 series de brácteas imbricadas, las brácteas medias oblongas, agudas u obtusas, de 2.1-3.4 mm de longitud y de 1.2-1.5 mm de ancho, endurecidas y engrosadas en la parte inferior, estrigosas, lanoso-pubescentes hacia el ápice herbáceo; flores liguladas 2 a 6, lígulas de 3.5-4 mm de longitud y 2-3 mm de ancho, con 7 a 9 nervaduras sobre la cara abaxial, amarillas, puberulentas, densamente cubiertas por gotas de resina; flores del disco 16 a 45, con lóbulos de 0.4-0.8 mm de longitud, amarillas, puberulentas, densamente cubiertas por gotas de resina; páleas abruptamente agudas; aquenios comprimidos o de sección subcuadrangular, los de las flores liguladas estériles, algo estriados, de 1.9-2.3 mm de longitud (¿depauperados?), glabros; vilano ausente.

Las plantas secas presentan un olor fétido, semejante al de basura en descomposición, el cual persiste por varios meses después de secado el material y va desapareciendo gradualmente.

TIPO: México, Durango, $\pm 1 \mathrm{~km}$ al NE de Las Ánimas, sobre la carretera a Nazas, municipio de Rodeo, 2508'36" N, 10425'59" W, 1340 m, arbusto muy abundante en pie de monte de lomerío, 5 Ago. 1997, S. González 5907, M. González y S. Acevedo (holotipo: IEB, isotipos: ANSM, CHAP, CHAPA, CIIDIR, ENCB, IBUG, MEXU, TEX).

Nombres comunes: chamuque, chaparro cenizo.

El epiteto de la especie nueva se acuña con el propósito de expresar reconocimiento a la labor realizada por el Instituto Nacional de Estadística, Geografía e Informática (INEGI). Como una de sus tareas principales, este organismo del gobierno federal de México tiene encomendada la elaboración de mapas del territorio de la República. Entre los diferentes tipos de documentos que ha publicado el INEGI a partir de 1970 (en otros tiempos también llamado CETENAL y DETENAL) destacan las "cartas de uso del suelo", que constituyen el resultado de una detallada cartografía de la vegetación del país, en variadas escalas.

Viguiera inegii parece estar relacionada con $V$. greggii por tener aquenios glabros y sin vilano y follaje e inflorescencias con gotas de resina evidentes, mientras que por la forma y pubescencia de las hojas y por el aspecto de las flores, se asemeja a $V$. brevifolia Greenm., especie cuya distribución geográfica general abarca el área donde $V$. inegii se desarrolla. De ambas especies, $V$. inegii difiere en tener hojas predominantemente alternas, con nervaduras del haz impresas y pubescencia más áspera, follaje de olor nauseabundo, cabezuelas y flores más pequeñas y pedúnculos más cortos. Otras diferencias pueden apreciarse en el Cuadro 1. Ocasionalmente presenta algunos capítulos anormales divididos en pequeñas cabezuelas aglomeradas, posiblemente reflejando introgresión genética de V. oligantha. 


\begin{tabular}{|c|c|c|c|c|c|c|c|c|c|c|}
\hline 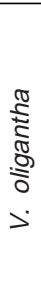 & 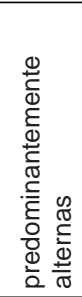 & 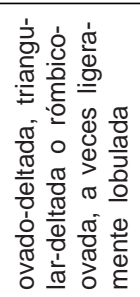 & 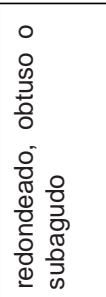 & 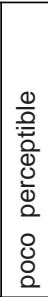 & 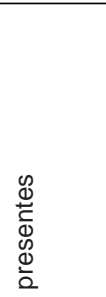 & 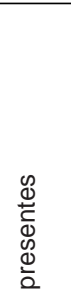 & 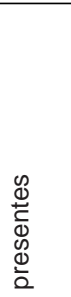 & 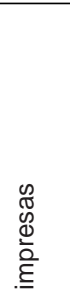 & 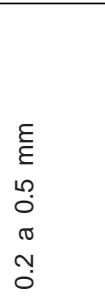 & 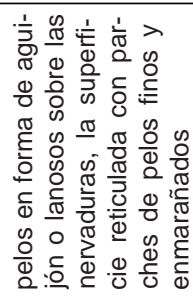 \\
\hline $\begin{array}{l}: \bar{D} \\
\stackrel{5}{\subseteq} \\
>\end{array}$ & 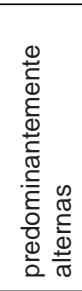 & 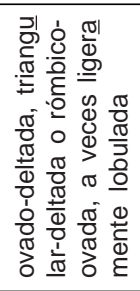 & 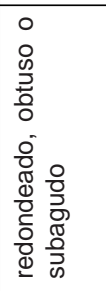 & 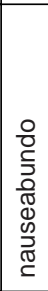 & 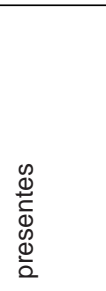 & 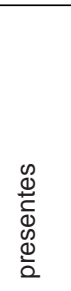 & 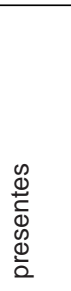 & 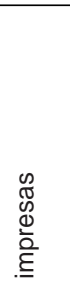 & 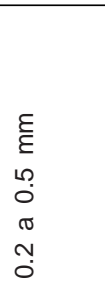 & 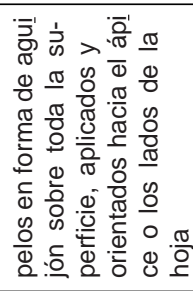 \\
\hline $\begin{array}{l}\text { : } \\
\text { ब } \\
\text { ప } \\
>\end{array}$ & $\begin{array}{l}\frac{9}{\pi} \\
\frac{\pi}{0} \\
\mathbb{\Phi} \\
\stackrel{0}{0}\end{array}$ & 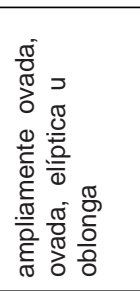 & 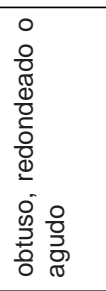 & $\begin{array}{l}\frac{0}{0} \\
\frac{\pi}{0} \\
\frac{\pi}{0} \\
\frac{\pi}{\pi}\end{array}$ & 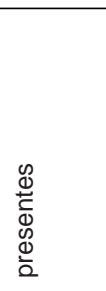 & 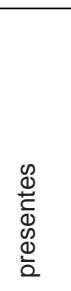 & 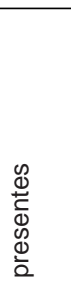 & 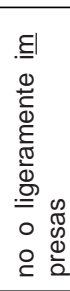 & 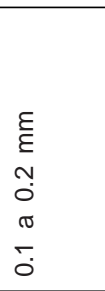 & 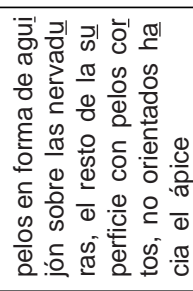 \\
\hline 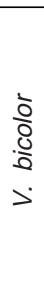 & $\begin{array}{l}\text { D } \\
\frac{\pi}{00} \\
\mathbb{\Phi} \\
\frac{0}{0}\end{array}$ & 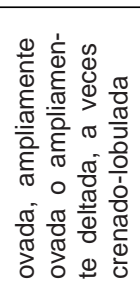 & 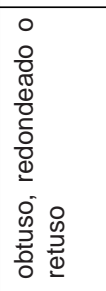 & 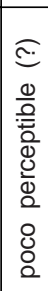 & 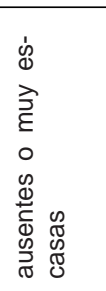 & 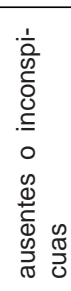 & 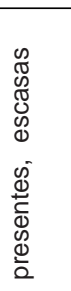 & 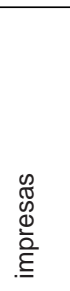 & 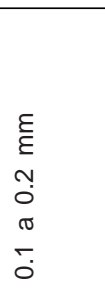 & 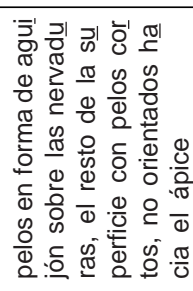 \\
\hline 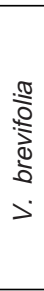 & 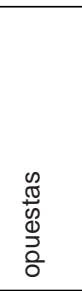 & 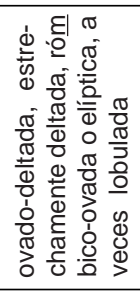 & 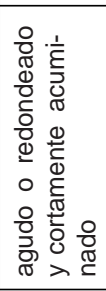 & 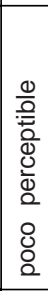 & 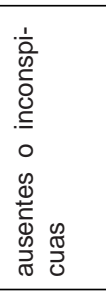 & 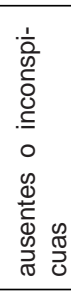 & 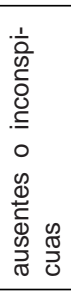 & 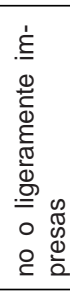 & 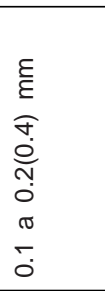 & 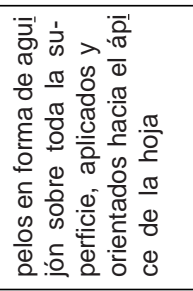 \\
\hline 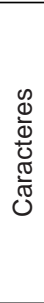 & $\begin{array}{l}\widehat{0} \\
: \frac{0}{0} \\
\frac{0}{0} \\
0 \\
\frac{0}{0} \\
\frac{0}{0} \\
0 \\
\frac{0}{0} \\
\frac{0}{1}\end{array}$ & 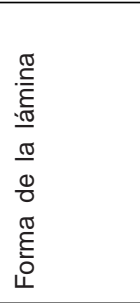 & 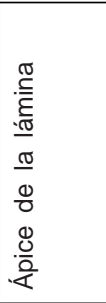 & $\begin{array}{l}\frac{\frac{0}{\sigma}}{\overline{\frac{\pi}{0}}} \\
\frac{0}{0} \\
\frac{0}{0} \\
\frac{0}{0}\end{array}$ & 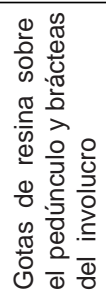 & 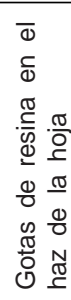 & 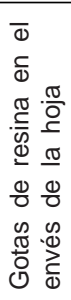 & 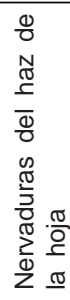 & 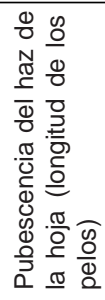 & 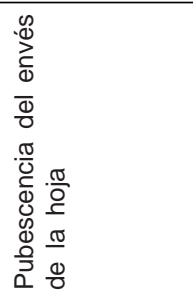 \\
\hline
\end{tabular}


González et al.: Nuevas Especies de Viguiera del Estado de Durango, México

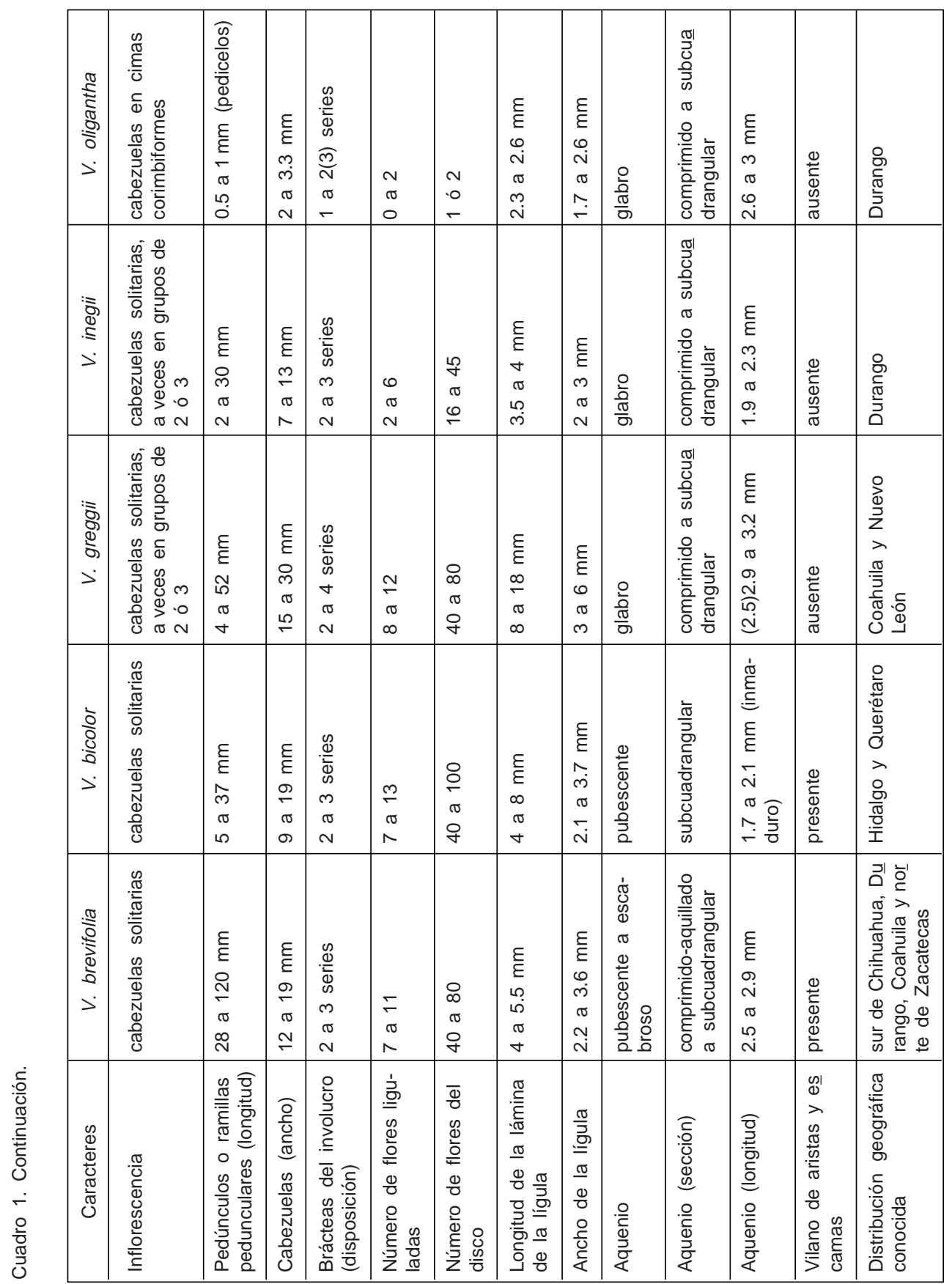


Viguiera inegii encuentra buen acomodo en la serie Brevifoliae (Blake, 1918) de la sección Viguiera (sección Chloracra S. F. Blake), junto con Viguiera greggii, V. brevifolia y $V$. bicolor S. F. Blake. La serie, conocida de México, incluye arbustos bajos muy ramificados, con tallos y hojas provistas de pubescencia canescente, aplicada; hojas opuestas, ovadas, canescentes al menos en el envés; cabezuelas solitarias en los extremos del tallo y ramas; involucro graduado de 3 a 4 series, las filarias ovadas u oblongo-ovadas, agudas, inconspicuamente costadas, con el ápice herbáceo muy corto e inconspicuo.

De acuerdo con la descripción anterior, $V$. inegii formaría parte de la serie Brevifoliae, pero no así $V$. oligantha. Esta última quedaría excluida debido a sus inflorescencias compuestas de muchas cabezuelas agrupadas en cimas corimbiformes en los extremos del tallo y las ramas (vs. solitarias), involucro reducido a 1 a 3 series (vs. 3 a 4 series), y pubescencia del envés de la hoja lanosa (vs. de pelos aplicados). Sin embargo, dado su evidente vínculo con $V$. inegii, a su vez relacionada con $V$. greggii y con $V$. brevifolia, se considera más adecuado ampliar la circunscripción de la serie Brevifoliae para incluir a $V$. oligantha, que establecer para esta última una serie separada.

Las características de la serie (incluyendo a $V$. oligantha) se amplían como sigue: arbustos bajos muy ramificados, con tallos y hojas con pubescencia canescente aplicada o lanosa; hojas orbiculares, elípticas, ovadas a deltadas, canescentes al menos en el envés; cabezuelas solitarias, en grupos de dos o tres, o bien, agrupadas en cimas corimbosas; involucro graduado de 2 a 4 series o a veces uniseriado, las filarias ovadas u oblongoovadas, agudas, inconspicuamente costadas, el ápice herbáceo muy corto e inconspicuo; vilano presente o ausente.

Las relaciones entre las tres especies anteriormente conocidas de Viguiera serie Brevifoliae (Viguiera greggii, V. brevifolia y $V$. bicolor) fueron estudiadas por Schilling y Panero (1988), quienes encontraron que la diferencia en composición de flavonoides está asociada con la presencia de tricomas glandulares subsésiles semejantes a gotas de resina, y que la composición de flavonoides indica que $V$. greggii y $V$. brevifolia son especies diferentes y no únicamente formas sin y con vilano de una misma entidad. Algunas diferencias adicionales entre $V$. greggii y $V$. brevifolia se presentan en el Cuadro 1.

En relación con este estudio el DNA de $V$. oligantha fue analizado por el Dr. J. L. Panero y la secuencia del ITS generada fue comparada por el Dr. E. Schilling con otras secuencias de la familia Compositae y particularmente de Viguiera. Los dos botánicos mencionados encontraron que $V$. oligantha está relacionada con $V$. ser. Brevifoliae pero tiene una rama filogenética muy larga, debido a muchos caracteres diferentes a los del resto de la serie, lo que hace pensar que las dos especies aquí descritas han divergido considerablemente de las otras tres del grupo.

Con el propósito de estudiar la ubicación taxonómica de los dos taxa que se proponen como nuevos, se realizaron muestreos y observaciones de plantas vivas, así como una revisión de materiales de herbario de los componentes de la serie Brevifoliae. En el Apéndice se presenta una relación de especímenes revisados de las tres especies anteriormente conocidas: V. bicolor, V. brevifolia y V. greggii. 


\section{CLAVE PARA LAS ESPECIES DE VIGUIERA SERIE BREVIFOLIAE}

1 Flores liguladas 0 a 2 por cabezuela, flores del disco 1 ó 2; cabezuelas agrupadas en cimas corimbiformes; pubescencia del envés de la hoja lanosa, los pelos enmarañados formando parches entre la reticulación de las venillas V. oligantha

1 Flores liguladas 2 a 13 por cabezuela, flores del disco 16 a 100; cabezuelas solitarias o en grupos de 2 ó 3; pubescencia del envés de la hoja de pelos rectos o algo curvados, pero no enmarañados formando parches entre la reticulación de las venillas ......... 2

2 Aquenio glabro; vilano ausente; tricomas glandulares (gotas de resina) evidentes sobre ambas superficies foliares

2 Aquenio pubescente o escabroso; vilano de aristas y escamas; tricomas glandulares (gotas de resina) ausentes o presentes en el haz, y ausentes o inconspicuos en el envés de la hoja

3 Hojas predominantemente alternas; follaje de olor nauseabundo; nervaduras del haz impresas; lámina de la lígula de $3.5-4 \mathrm{~mm}$ de largo y de $2-3 \mathrm{~mm}$ de ancho

Hojas opuestas; follaje impresas; lámina de la lígula de $8-18 \mathrm{~mm}$ de largo y de 3-6 $\mathrm{mm}$ de ancho

V. greggii

4 Pedúnculos o ramillas pedunculares de 5-37 mm de largo; láminas de las hojas ovadoorbiculares a orbiculares, obtusas a redondeadas o retusas en el ápice; tricomas glandulares (gotas de resina) presentes sobre el envés de la hoja; plantas conocidas de Querétaro e Hidalgo V. bicolor

4 Pedúnculos o ramillas pedunculares de 28-120 mm de largo; láminas de las hojas ovadodeltadas, estrechamente deltadas o rómbico-ovadas, agudas a redondeadas y cortamente acuminadas en el ápice; tricomas glandulares (gotas de resina) ausentes o inconspicuas en ambas superficies foliares; plantas conocidas del sur de Chihuahua al norte de Zacatecas V. brevifolia

\section{AGRADECIMIENTOS}

Damos las gracias a los señores Roberto y Noé González Elizondo por el apoyo logístico para colectas de material de $V$. greggii de Nuevo León y Coahuila y a Roberto González, Dora M. Leal y Francisco Estrada por participar en las colectas. A los Ing. Jorge García y Víctor López, del INEGI, quienes colectaron material de V. oligantha. AI Dr. J. L. Panero por analizar el DNA de $V$. oligantha y al Dr. E. Schilling por la comparación de éste con otras secuencias de compuestas. A los M. en C. Jorge A. Tena, Irma Lorena López y M. A. Márquez por su apoyo en diversas fases del trabajo y al Dr. A. A. Reznicek por facilitar literatura. Agradecemos a los encargados de los herbarios ANSM, CHAPA, CIIDIR, ENCB, IEB y MEXU por las facilidades brindadas y a dos revisores anónimos por sus valiosas sugerencias y comentarios al manuscrito. 


\section{LITERATURA CITADA}

Blake, S. F. 1918. A revision of the genus Viguiera. Contr. Gray Herb. 54: 1-205.

Bravo-Hollis, H. y H. Sánchez Mejorada. 1991. Las cactáceas de México. Vol. III. Universidad Nacional Autónoma de México. México, D.F. 643 pp.

Henrickson, J. y R. A. Hilsenbeck. 1979. New taxa and combinations in Siphonoglossa (Acanthaceae). Brittonia 31(3): 373-378.

Iltis, H. H. 1999. Setchellanthaceae (Capparales), a new family for a relictual, glucosinolate-producing endemic of the Mexican deserts. Taxon 48: 257-288.

Rzedowski, J. 1972. Contribuciones a la fitogeografía florística e histórica de México. III. Algunas tendencias en la distribución geográfica y ecológica de las Compositae mexicanas. Ciencia, Méx. 27: $123-132$.

Rzedowski, J. y G. Calderón de Rzedowski. 1999. Hallazgo de plantas mexicanas del género Cotinus (Anacardiaceae) con características de reliquias del Terciario. Acta Bot. Mex. 47: 23-30.

Turner, B. L. 1977. Henricksonia (Asteraceae-Coreopsidinae), a newly discovered genus with a paleaceous pappus from North-central México. Amer. J. Bot. 64(1): 78-80.

Schilling, E. E. y J. L. Panero. 1988. Flavonoids of Viguiera series Brevifoliae. Biochem. Syst. Ecol. 16(4): 417-418.

Villarreal, J. A. 1992. Dos nuevos taxa del género Mimosa (Leguminosae: Mimosoideae) para el norte de México. Acta Bot. Mex. 20: 46. 
González et al.: Nuevas Especies de Viguiera del Estado de Durango, México

\section{APÉNDICE}

Especímenes revisados de Viguiera greggii, $V$. brevifolia y $V$. bicolor.

Viguiera greggii (A. Gray) S. F. Blake

COAHUILA: Sierra de La Paila (lado norte), cañada Becerros, 101042' N, 261' W, J. A. Villarreal 5439, M. A. Carranza y L. Arce (ANSM, CHAPA); hills near Saltillo, C. G. Pringle 8800 (ENCB, MEXU); El Carmen, carr. $57 .$, aprox. $25 \mathrm{~km}$. N de Saltillo, área cercana al entronque, J. A. Villarreal y M. A. Carranza 7915 (ANSM, MEXU); Cañón de San Lorenzo, al sur de Saltillo, L. Arce s.n. (CIIDIR); Carneros Pass, C. G. Pringle 2387 (MEXU); Paso de Carneros, D. S. Seigler y M. Aregullin DS 11484 (MEXU); aprox. $1 \mathrm{~km}$ al W de Estación Benjamín (Carneros), entronque a carretera 54 (Saltillo-Concepción del Oro), sobre el camino a la torre de microondas, mpio. Saltillo, 2507'20" N, 10107'05" W, S. González 6094 con R. González, D. Leal, F. Estrada (ANSM, CIIDIR, ENCB, IEB, MEXU, TEX); Estación de Carneros, carr. a Zacatecas, $30 \mathrm{Km}$ al $\mathrm{S}$ de Saltillo, $25^{\circ} 07^{\prime} \mathrm{N}, 101^{\circ} 07^{\prime} \mathrm{W}$, J. Valdés, M. A. Carranza C-450 (ANSM, CHAPA, CIIDIR); Estación de microondas Carneros, aprox. $2.5 \mathrm{~km}$ al W de la estación de ferrocarril Carneros, $32 \mathrm{~km}$ al $S$ de Saltillo por la carretera a Zacatecas, 2507'09" N, 10107'09" W, J. L. Villaseñor 1290 con J. I. Calzada (MEXU); mpio. Saltillo, Rancho Los Angeles, $48 \mathrm{Km}$ al S de Saltillo, carr. Saltillo-Concepción del Oro (54), 260ㅜ N, 101066' W, M. A. Carranza 464, J. Valdés (ANSM, CIIDIR (2)); mpio. Saltillo, Rancho Los Ángeles, $3.2 \mathrm{~km}$ del entronque a carretera 54 (Saltillo-Concepción del Oro), y aprox. $1 \mathrm{~km}$ al SW del camino al rancho, 2508'19" N, 101'04'24" W, S. González 6095 con R. González, D. Leal, F. Estrada (ANSM, CIIDIR, ENCB, IEB, MEXU, TEX); Rancho demostrativo Los Ángeles, $48 \mathrm{~km}$ al sur de Saltillo, D. Arredondo 100 (CHAPA); 39 mi north of Concepción del Oro, J. Youngpeter \& T. J. Cohn 54 (ENCB); Cuevecillas, Arteaga, D. Fuentes s.n. (ENCB); Puerto de las Flores, mpio. de Arteaga, M. F. Robert 187 (ENCB); 22 millas al W de Ocampo hacia la cuesta Zozoya, P. Peterson y J. Valdés 2000 (CHAPA (2)).

NUEVO LEÓN: 5 miles south of the Coahuila line on the main Saltillo-Matehuala highway, M. C. Johnston 5866 (MEXU); Galeana, 4 km N Potosí, Hinton et al. 18774 (CIIDIR, ENCB, MEXU); Galeana, $4 \mathrm{~km}$ al $\mathrm{N}$ de El Potosí, por el camino a San José de La Joya, 2452'38" N, 100¹9'02" W, S. González 6096 con R. González, D. Leal, F. Estrada (ANSM, CIIDIR, ENCB, IEB, MEXU, TEX); Rancho Aguililla, Galeana, Hinton et al. 19730 (CHAPA); Galeana, SW slope Potosí, Hinton et al. 18567 (CIIDIR); Galeana, SW slope Potosí, Hinton et al. 18568 (ENCB); Los Sauces, Galeana, Hinton et al. 18094 (ENCB-3 ejemplares); Galeana, Santa Rita de Cordeladas, ca. 7 mi NE of San Roberto junction (Hwys. 57 \& 31), low gypsum hills S of Cerro Potosí, 2445' N, 100'12' W, S. Sundberg et al. 1812 (MEXU).

Viguiera brevifolia Greenm.

CHIHUAHUA: $14 \mathrm{~km}$ SE of Rancho La Gloria, on the road to Cerros Blancos, 27015'40" N, 10409' W, F. Chiang, T. Wendty M. C. Johnston 9092 (MEXU); Jiménez, central

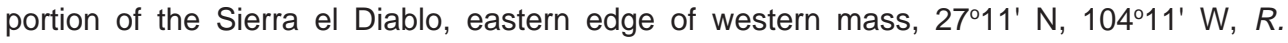
Spellenberg y M. Mahrt 10730 (CIIDIR).

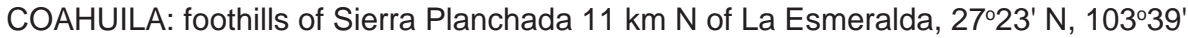
W, M. C. Johnston, T. Wendt y F. Chiang 10874 (MEXU); Sierra de Jimulco, mina de San 
José, hacia la cima, 2508' N, 10313' N, M. A. Carranza 1924 (ANSM, IEB); Parras, Hinton et al. 23536 (IEB); S. Paila (C. Carrera), distr. G. Cepeda, Hinton et al. 16583 (IEB).

DURANGO: km 195 del tramo La Zarca-Durango, E. García M. 409 (CHAPA, CIIDIR); Rodeo, al N, km 195.3 de carretera Durango-Parral, tramo Durango-La Zarca, 25²6'29" N, 104³6'01" W, S. González 6196 con M. A. Márquez (CIIDIR); Puente de Hojuela, 8 km al SE de Mapimí, E. Torrecillas 243 (MEXU); $71 \mathrm{~km} \mathrm{~W} \mathrm{of} \mathrm{Bermejillo,} \mathrm{H.} \mathrm{S.} \mathrm{Gentry} 6836$ (MEXU); Cuencamé, J. Marroquín 2602 (ENCB).

ZACATECAS (límite con Coahuila): $10 \mathrm{~km}$ al E de Melchor Ocampo, sobre el camino a Concepción del Oro, J. Rzedowski 9324 (ENCB).

Viguiera bicolor S. F. Blake

QUERÉTARO: $9.3 \mathrm{~km}$ al E de Buenavista, municipio de Cadereyta, S. Zamudio 3290 (IEB, MEXU); $4 \mathrm{~km}$ al NE de San Javier Las Tuzas, municipio de Cadereyta de Montes, S. Zamudio 2738 (IEB, MEXU); Mesa de León, municipio de Cadereyta, Z. Ortega 503 (IEB).

HIDALGO: $8 \mathrm{~km}$ al $\mathrm{N}$ de Zimapán, municipio de Zimapán, R. Hernández 3678 (MEXU); $20.4 \mathrm{~km}$ east of Zimapan, dirt road to mines, 2040' N, 99 $30^{\prime} \mathrm{W}$, A. Prather 936b with J. Soule (MEXU); Cerro Boludo, Barranca de Tolantongo, municipio de Cardonal, $P$. Hiriart et al. 101 (MEXU); Barranca de Metztitlán, cerca del puente de Venados, J. Rzedowski 19526 (MEXU); Mezquititlán, Venados, 3-4 km al NE de Venados, 20²8'15" N, 9840'07" W, 1400 m, F. González M. 4900 con R. Dirzo y R. M. López (MEXU); laderas al W del entronque de la carretera a Metztitlán con la carretera Pachuca-Tampico, J. L. Villaseñor 132, F. Chiang y A. Valiente (MEXU); $1.5 \mathrm{~km}$ al $\mathrm{W}$ de Huizache, mpio. Caltimacán, G. Asteinza B. 562-12 (ENCB); La Lomita al S de Tasquillo y al NW de Danghú, municipio de Tasquillo, P. Tenorio 506 y C. Romero de T. (MEXU (2)); kilómetro 172 carretera MéxicoLaredo, Valle del Mezquital, año 1936, sin colector 9197 (MEXU). 